\title{
Effective site-energy model: A thermodynamic approach applied to size-mismatched alloys
}

\author{
F. Berthier,,$^{1,2, *}$ J. Creuze, ${ }^{2}$ and B. Legrand ${ }^{3}$ \\ ${ }^{1}$ Centre National de la Recherche Scientifique (CNRS), Unité mixte de recherche (UMR) 8182, F91405 Orsay Cedex, France \\ ${ }^{2}$ Synthèse, Propriétés \& Modélisation des Matériaux (SP2M)/Institut de Chimie Moléculaire et des Matériaux d'Orsay (ICMMO), \\ Université Paris Sud, UMR 8182, Université Paris-Saclay, F91405 Orsay Cedex, France \\ ${ }^{3}$ DEN-Service de Recherches de Métallurgie Physique, CEA, Université Paris-Saclay, F91191 Gif-sur-Yvette Cedex, France
}

(Received 7 February 2017; published 8 June 2017)

\begin{abstract}
We present a novel energetic model that takes into account atomistic relaxations to describe the thermodynamic properties of $A_{c} B_{1-c}$ binary alloys. It requires the calculation of the energies on each site of a random solid solution after relaxation as a function of both the local composition and the nominal concentration. These site energies are obtained by molecular static simulations using $\mathrm{N}$-body interatomic potentials derived from the second-moment approximation (SMA) of the tight-binding scheme. This new model allows us to determine the effective pair interactions (EPIs) that drive the short-range order (SRO) and to analyze the relative role of the EPIs' contribution to the mixing enthalpy, with respect to the contribution due to the lattice mismatch between the constituents. We apply this formalism to Au-Ni and Ag-Cu alloys, both of them tending to phase separate in the bulk and exhibiting a large size mismatch. Rigid-lattice Monte Carlo (MC) simulations lead to phase diagrams that are in good agreement with both those obtained by off-lattice SMA-MC simulations and the experimental ones. While the phase diagrams of $\mathrm{Au}-\mathrm{Ni}$ and $\mathrm{Ag}-\mathrm{Cu}$ alloys are very similar, we show that phase separation is mainly driven by the elastic contribution for Au-Ni and by the EPIs' contribution for Ag-Cu. Furthermore, for Au-Ni, the analysis of the SRO shows an inversion between the tendency to order and the tendency to phase separate as a function of the concentration.
\end{abstract}

DOI: 10.1103/PhysRevB.95.224102

\section{INTRODUCTION}

The ability to predict and to understand solid phase diagrams of alloys is still of great interest in metallurgy. In particular, research in solidification, crystal growth, and phase transformation are based on the knowledge of phase diagrams. Phase diagrams are also of great interest in technology, the knowledge of a full and complete description of the stable phases as a function of composition and temperature being essential to achieve the desired microstructure. From a more fundamental point of view, one of the remaining difficulties is to account for both ordering phenomena [characterized by the short-range (SRO) and long-range (LRO) orders] and atomic relaxations (depending themselves on the degree of order), especially for alloys presenting a large size mismatch between the constituents. Therefore, the understanding of the interplay between chemical-type interactions and elastic-type ones is still challenging, as are their consequences on phase diagrams.

At the atomic scale, numerous methods have been developed in order to compute equilibrium phase diagrams. They can be grouped into two main families, those using a rigid-lattice formalism and those involving off-lattice simulations, both of them using energetic models at different levels of empiricism. Monte Carlo (MC) simulations using semiempirical interatomic potentials are the most common off-lattice simulations [1-9]. The analysis of the driving forces is rarely done, the authors focusing on the phase diagram or the segregation isotherms resulting from the potential. Analysis by an effective Ising model treated in a mean-field approximation (MFA) proved to be very efficient in dealing with the coupling between chemical order and atomic displacements [8]. At a more precise level of description of the electronic structure,

*Corresponding author: fabienne.berthier@u-psud.fr traditional first principles calculations excel at providing formation energies at absolute zero, but obtaining thermodynamic information at nonzero temperatures requires suitable sampling of all the excited states visited in thermodynamic equilibrium, which is computationally prohibitive via bruteforce quantum mechanical calculations alone. In this context, $a b$ initio methods use a rigid-lattice approach such as the generalized perturbation model (GPM) with respect to the mean-field coherent potential approximation (CPA) [10,11] or the cluster expansion (CE) formalism to address this issue [12-26]. The GPM is used to determine the effective cluster interactions (ECIs) which are defined in the totally disordered alloy characterized by the CPA. The CE is based on the calculation of the energy of many ordered phases at different concentrations leading also to ECIs which depend on the concentration. Then the phase diagram is obtained by calculating the free energy via the cluster variation method [27] or via MC simulations and by taking into account the vibrational entropy, at least in the most recent studies [28,29]. To account not only for the chemical effects of local ordering but also for the effects of atomic relaxations in size-mismatched alloys, the mixed-space CE has also been developed [21]. It introduces an additional term in the $\mathrm{CE}$, which corresponds to an infinite range of real-space elastic interaction terms [21]. In parallel to these developments involving first principles calculations, Asta and Foiles have proposed another method, SOE-EAM, to take advantage of both the semiempirical level of description of the electronic structure and the rigid-lattice simulations speed [30]. It consists of combining a second-order expansion (SOE) formalism with a description of the energy of alloys based on the embedded atom method (EAM) to take into account the atomic displacements together with a quasiharmonic approximation to evaluate the vibrational entropy. The SOE of the energy allows the calculations of 
effective pair interactions (EPIs) which are then used in MC simulations on rigid lattice with the addition of a vibrational entropy term or in a mean-field modeling.

In this paper, we present the effective site energies (ESEs) model, a novel method to depict the thermodynamic properties of $A_{c} B_{1-c}$ binary alloys, in particular the phase diagrams and SRO. This method allows for the analysis of the driving forces (phase diagrams, equilibrium segregation), and it can be developed from any energetic model giving access to the site energies. Sophisticated tight-binding approaches, such as the Naval Research Laboratory tight-binding NRL-TB method and even recent schemes in $a b$ initio calculations, can provide the onsite energies, but this issue is not common and remains a challenge for the latter since the projection mode does not enable one to define site energies unambiguously [31,32]. This method is thus mainly adapted to any semiempirical potential (Lennard-Jones, EAM, tight-binding in the second moment approximation [SMA], in the fourth moment approximation [FMA], or other). Up to now, these site energies have not yet been analyzed in detail or used in a thermodynamic modeling.

The main idea is to separate the dependency of the site energies on the local environment (the definition of the latter is to be specified) from the dependency on the nominal concentration that reflects a nonlocal dependency. Thus, in the absence of magnetism and fine details of the electronic densities of state, the concentration dependency of the site energies is mainly related to the elastic effects as, for example, their dependency on the lattice parameter.

The site energies are obtained in a reference state selected as a fully random solid solution (rss). Then standard relaxation algorithms at $T=0 \mathrm{~K}$ allow one to obtain these energies taking into account relaxation of the atomic positions. In this paper, we use interatomic potentials derived from the SMA of the tight-binding scheme. The site energies $E_{I}^{p}(c)$ $(I=A, B)$ are obtained for both a given local surrounding, defined for example by the number $p$ of $A$ atoms in the nearest-neighboring shell, and a given nominal concentration $c$. The mathematical description of a rss leads to analytical expressions of the relevant thermodynamic quantities of the alloy (mixing enthalpy, permutation enthalpy) as a function of the site energies on the whole range of nominal concentration. Hence, the ESE model is a useful theoretical tool to separate the effects of the local chemical environment from those of the global composition. Using the site energies in rigid-lattice MC simulations or in a mean-field modeling has the advantage of being faster than off-lattice simulations while taking into account the effects of atomic relaxations and the influence of both the local composition and the nominal concentration.

Moreover, in order to make the link with the more classical Ising-type formulations of alloy thermodynamics, we have made a mapping between the ESE model and a generalized Ising model based on EPIs. From this mapping, the analysis of the permutation enthalpy (the enthalpy change when turning an atom B to A), which is the key quantity in the alloys thermodynamics $[33,34]$, allows one to define the contributions related to local effects (so-called chemical effect) and to nonlocal effects (so-called size effect). The chemical effect is related to the EPIs that drive SRO, and the size effect is directly related to the size mismatch between the constituents. A deep analysis of these contributions on the whole range of concentration has not yet been carried out, even if major achievements have already been made in the infinite dilute limits $[35,36]$. Furthermore, we show how to determine the vibrational entropy of permutation from a mean-field analysis of the off-lattice MC isotherms and how it can be easily introduced into the ESE formalism.

We apply this formalism to two alloys characterized by a large size mismatch between the constituents and a phase diagram with a large miscibility gap: $\mathrm{Au}-\mathrm{Ni}$ and $\mathrm{Ag}-\mathrm{Cu}$. $\mathrm{Au}-\mathrm{Ni}$ has received a lot of attention, both experimentally and theoretically, especially due to a specific behavior of the SRO in this system [12,18,37-45]. Ag-Cu has also been frequently studied, both in the bulk and at interfaces [2,8,13,14,46-53], and the two systems have been compared theoretically in a few studies $[21,22,30,48]$. Therefore, the aims of this paper are first to test the method (the definition of the local environment, for example) and to obtain an analysis of the driving forces of the thermodynamics of these two alloys. Thanks to the ESE model, we show that the relative weight of the two contributions described above leads to very different behaviors for both the SRO in rss and the relative stabilities between incoherent phase separation, rss, and coherent phase separation as the thermodynamic equilibrium state [48].

This paper is organized as follows. Section II introduces the ESE model, the Hamiltonian (Sec. II A), the procedure to get the site energies (Sec. II B), the rigid-lattice MC simulations, and the mean field approach based on this energetic model (Sec. II C), and the mapping with a generalized Ising model (Sec. IID). Section III is devoted to the results obtained for $\mathrm{Au}-\mathrm{Ni}$ and $\mathrm{Ag}-\mathrm{Cu}$ alloys: the site energies are analyzed (Sec. III A), and their use to describe accurately the energetics of rss is validated (Sec. III B). Then we analyze the differences between $\mathrm{Au}-\mathrm{Ni}$ and $\mathrm{Ag}-\mathrm{Cu}$ alloys (Sec. III C). Isotherms and phase diagrams are, respectively, presented in Secs. IIID and IIIE. Finally, in Sec. IV, we discuss the capabilities of the ESE model (Sec. IV A) and the main features of the thermodynamics of the considered size-mismatched alloys (Sec. IV B).

\section{ESES MODEL}

\section{A. Hamiltonian}

The ESE model is based on the determination of the site energy $E_{I}^{p}(c)$ of an atom $\mathrm{I}(I=A, B)$ as a function of the number $p$ of $A$ nearest-neighbors in a relaxed rss of a $A_{c} B_{1-c}$ binary alloy. Only the nearest-neighbor shell is considered in this paper since a good agreement with the SMA simulations is obtained for the two systems (see Sec. III B). However, note that the formalism presented hereafter can be extended to more distant shells of neighbor.

The Hamiltonian $H$ of a rss binary alloy composed of $N_{A}$ atoms $A$ and $N_{B}$ atoms $B$ can be written in the following form:

$$
H=\sum_{i=1}^{N_{A}} E_{A}^{p_{i}}(c)+\sum_{j=1}^{N_{B}} E_{B}^{p_{j}}(c),
$$

with $p_{i}$ (respectively, $p_{j}$ ) the number of $A$ atoms in the nearestneighbor shell of the $i$ th $A$ atom (respectively, $j$ th $B$ atom). 
As we consider a rss, the Hamiltonian can be rewritten using a combinatorial description

$$
H(c)=N_{\mathrm{at}} \sum_{p=0}^{Z} C_{Z}^{p} c^{p}(1-c)^{Z-p}\left(c E_{A}^{p}(c)+(1-c) E_{B}^{p}(c)\right),
$$

with $N_{\text {at }}$ the total number of atoms, $c=N_{A} / N_{\text {at }}$ the nominal concentration and $Z$ the number of nearest neighbors. Here, $C_{Z}^{p}$ are the binomial coefficients, i.e. the number of ways that $p A$ neighbors can be chosen among $Z$ neighbors disregarding their order.

The mixing enthalpy $\Delta H^{\text {mixing }}$ corresponds to the formation energy of a rss, and its sign gives the tendency of the alloy to form homoatomic or heteroatomic bonds. In the ESE model, $\Delta H^{\text {mixing }}$ is given by

$$
\Delta H^{\text {mixing }}(c)=H(c) / N_{\mathrm{at}}-\left[c E_{A}^{Z}(1)+(1-c) E_{B}^{0}(0)\right],
$$

where $E_{A}^{Z}(1)=E_{\mathrm{coh}}^{A}$ and $E_{B}^{0}(0)=E_{\mathrm{coh}}^{B}, H(c)$ being given by Eq. (2). Positive values of $\Delta H^{\text {mixing }}$ indicate a tendency to phase separate.

\section{B. Computational procedure}

We use $N$-body interatomic potentials derived from the SMA of the local density of states within the tight-binding scheme [54] to compute $E_{I}^{p}(c)$. Parameters of these potentials are given in Table I. These potentials have been shown to be quite successful to study bulk, surface, and grain boundaries in metallic alloys [8,49-52,55]. The FIRE algorithm [58] is used to minimize the potential energy of the rss at $T=0 \mathrm{~K}$. The simulation box is constructed with 7 face-centered-cubic (fcc) cells in each direction $\left(N_{\text {at }}=1372\right.$ atoms $)$ and threedimensional (3D) periodic boundary conditions are used. The volume of the simulation box is optimized for each concentration $c$. Then the procedure can be described as follows:

(i) a site $i$ is selected, and its chemical nature is fixed ( $I=A, B)$;

(ii) the local environment of $i$ is constructed randomly with $p$ nearest neighbors being $A$ atoms, the remaining $12-p$ sites being filled with $B$ atoms;

(iii) the remaining $N_{\mathrm{at}}-13$ sites are filled randomly with $A$ and $B$ atoms to reach the nominal concentration $c$.

For a given rss, the $E_{I}^{p}(c)$ are averaged over all the existing configurations surrounding atoms of type $I$ that correspond with the local $p$ environment in $A$ atoms. Finally, the procedure is repeated 100 times for each local $p$ environment to obtain good statistics over the $E_{I}^{p}(c)$.

\section{ESE simulations}

To study the thermodynamics of a $A_{c} B_{1-c}$ binary alloy, the ESEs can then be used either in a MFA (ESE-MFA) or in rigid-lattice $\mathrm{MC}$ simulations on (ESE-MC) to go beyond the MFA.

\section{ESE-MFA}

For an Ising model treated in the MFA (see Appendix A), the minimization of the grand canonical free energy with respect to the nominal concentration $c$ leads to the bulk isotherm at temperature $T[8]$

$$
\frac{c}{1-c}=\exp \left(-\frac{\Delta H^{\text {perm }}-\Delta \mu}{k_{B} T}\right),
$$

with $\Delta \mu=\mu_{A}-\mu_{B}$, the difference in chemical potentials between pure metals $A$ and $B[59,60]$, and $\Delta H^{\text {perm }}$ the permutation enthalpy, i.e., the enthalpy change of the system when turning a $B$ atom into an $A$ atom. Following Eq. (4), $\Delta H^{\text {perm }}$ is the relevant quantity that controls the chemical equilibrium configuration, and it is obtained from the derivation of the total enthalpy of the system with respect to $c$ (see Appendix A). In the ESE approach [Eq. (1)], $\Delta H^{\text {perm }}$ is composed of three terms

$$
\Delta H^{\text {perm }}(c)=\Delta H_{B \rightarrow A}(c)+\Delta H_{n}(c)+\Delta H_{\Delta c}(c),
$$

where

(1) $\Delta H_{B \rightarrow A}$ is the enthalpy change on the site where the exchange $B \rightarrow A$ occurs

$$
\Delta H_{B \rightarrow A}=H_{A}-H_{B} ;
$$

(2) $\Delta H_{n}$ is the enthalpy change on all nearest-neighbors sites of the one on which the exchange $B \rightarrow A$ occurs

$$
\Delta H_{n}=\sum_{p=0}^{Z} C_{Z}^{p} \frac{\partial\left[c^{p}(1-c)^{Z-p}\right]}{\partial c}\left[c E_{A}^{p}(c)+(1-c) E_{B}^{p}(c)\right]
$$

TABLE I. Parameters of the SMA potentials for the Au-Ni and Ag-Cu alloys. The total energy $E_{i}$ on each site $i$ is written as $E_{i}=$

\begin{tabular}{|c|c|c|c|c|c|c|c|c|c|}
\hline & $A(\mathrm{eV})$ & $p$ & $\xi(\mathrm{eV})$ & $q$ & $B(\mathrm{GPa})$ & $C_{44}(\mathrm{GPa})$ & $C^{\prime}(\mathrm{GPa})$ & $E_{\text {coh }}(\mathrm{eV} / \mathrm{at})$ & $r_{0}(\AA)$ \\
\hline $\mathrm{Ni}-\mathrm{Ni}$ & 0.1217 & 10.7626 & 1.6396 & 2.435 & $186(188)$ & 97 (132) & $29(55)$ & $-4.44(-4.44)$ & 2.49 \\
\hline $\mathrm{Au}-\mathrm{Au}$ & 0.2134 & 10.4201 & 1.8303 & 4.1765 & $173(165)$ & $43(42)$ & $16(15)$ & $-3.81(-3.81)$ & 2.885 \\
\hline $\mathrm{Ni}-\mathrm{Au}$ & 0.1555 & 10.5914 & 1.7149 & 3.3057 & & & & & 2.6875 \\
\hline $\mathrm{Cu}-\mathrm{Cu}$ & 0.1084 & 10.377 & 1.3434 & 2.6335 & $141(142)$ & $73(82)$ & $21(26)$ & $-3.5(-3.5)$ & 2.56 \\
\hline $\mathrm{Ag}-\mathrm{Ag}$ & 0.1249 & 10.3453 & 1.2672 & 3.4236 & $111(108)$ & $43(52)$ & $15(16)$ & $-2.95(-2.95)$ & 2.89 \\
\hline $\mathrm{Cu}-\mathrm{Ag}$ & 0.1188 & 10.3612 & 1.2996 & 3.0286 & & & & & 2.725 \\
\hline
\end{tabular}
$-\sqrt{\sum_{j} \xi_{I J}^{2} \exp \left[-2 q_{I J}\left(r_{i j} / r_{0}^{I J}-1\right)\right]}+\sum_{j} A_{I J} \exp \left[-p_{I J}\left(r_{i j} / r_{0}^{I J}-1\right)\right]$, where $r_{i j}$ is the distance between atoms of type $I$ and $J(I, J=A, B)$ at sites $i$ and $j$, respectively, and $r_{0}^{I J}$ is the nearest-neighbor distance in the metal $\left.I\left(r_{0}^{I J}=\left(r_{0}^{I I}+r_{0}^{J J}\right) / 2\right)\right)$. We ensure the continuity in the computation of energies and forces by shrinking the exponentials to zero via a fifth-order polynomial between $r_{i j}=\max \left(\sqrt{2} r_{0}^{I I}, \sqrt{2} r_{0}^{J J}\right)$ and $r_{i j}=\min \left(2 r_{0}^{I I}, 2 r_{0}^{J J}\right)$. The experimental values are indicated in parentheses and are taken from Ref. [56] for $E_{\text {coh }}$ and $r_{0}$, and from Ref. [57] for $B, C_{44}$, and $C^{\prime}$. 
(3) $\Delta H_{\Delta c}$ is a complementary term related to the variation of the site energies for all sites due to the change in nominal concentration induced by the exchange $B \rightarrow A$

$$
\begin{aligned}
\Delta H_{\Delta c}= & N_{\text {at }} \sum_{p=0}^{Z} C_{Z}^{p} c^{p}(1-c)^{Z-p} \\
& \times\left[c \frac{\partial E_{A}^{p}(c)}{\partial c}+(1-c) \frac{\partial E_{B}^{p}(c)}{\partial c}\right] \Delta c \text { with } \\
\Delta c= & 1 / N_{\text {at }} .
\end{aligned}
$$

Thus, using Eqs. (6)-(8), the ESE model leads to an analytical formula of the permutation enthalpy [Eq. (5)] for a rss on the whole range of nominal concentration $c$. This formula can be introduced in the ESE-MFA formalism via Eq. (4) to obtain isotherms at different temperatures.

\section{ESE-MC simulation in the canonical ensemble}

To avoid the assumption of a homogeneous concentration, we also perform equilibrium MC simulations in the canonical ensemble starting from the same Hamiltonian, Eq. (1). In this ensemble, the nominal concentration $c$ and the temperature $T$ are fixed. Using the Metropolis algorithm to generate a series of atomic configurations [61], one event consists of exchanging the positions of two atoms $A$ and $B$ randomly selected. The probability for accepting the configurational change is $P=\min \left[1, \exp \left(-\Delta E / k_{B} T\right)\right]$, where $\Delta E$ is the change in internal energy between the initial and final states. One MC step consists in the proposal of an exchange for each of the minority atoms. At each step, the difference in chemical potentials $\Delta \mu$ is determined via Widom's method [62] to get the isotherm $\Delta \mu(c)$. To characterize the chemical local order, the number of heteroatomic nearest-neighbor bonds is evaluated at each step. The thermodynamical quantities are averaged over $510^{3} \mathrm{MC}$ steps.

\section{Relation between the Ising and ESE models}

The matching with the Ising model [Eqs. (A9) or (A13) via the MFA] is an efficient way to check the consistency of this new approach and to analyze the results. This requires the derivation of the relevant quantities occurring in the permutation enthalpy, namely $\tau(c)$ and $V(c)$, from the site energies. Here, $Z \tau(c)$ is defined as

$$
Z \tau(c)=E_{A}^{Z}(c)-E_{B}^{0}(c),
$$

where $E_{A}^{Z}(c)$ [respectively, $E_{B}^{0}(c)$ ] is the site energy of an $A$ (respectively, $B$ ) atom surrounded by $Z$ atoms $A$ (respectively, $\mathrm{Z}$ atoms $B$ ) in a $A_{c} B_{1-c}$ rss. For constant nearest-neighbor pair interactions, Eq. (9) provides $Z \tau(c)=Z\left(V_{1}^{A A}-V_{1}^{B B}\right) / 2$, which is equal to the standard difference in cohesive energies between pure metals, consistent with Eq. (A9). In the general case, $Z \tau(c)$ can be understood as follows: $E_{A}^{Z}(c)$ and $E_{B}^{0}(c)$ are the site energies of a central atom $I(=A, B)$ in a cluster of $Z+1$ atoms $I(=A, B)$ immersed in a relaxed rss of concentration $c$. Then the physical origin of $Z \tau(c)$ is mainly related to the variation of the rss lattice parameter as a function of the concentration.

The alloy pair interactions are obtained by considering the enthalpy difference of a system containing two atoms $A$ apart from one another (initial state) and two atoms $A$ in $R$ th neighbor positions (final state) in a rss at a given concentration [35]. Up to now, EPIs have been determined directly from simulations only in the infinite dilute limits $[35,36]$. The ESE model allows one to get an analytical expression of the EPIs on the whole range of nominal concentration.

First, we evaluate the energy balance restricted to the nearest-neighbor shell. Here, $V_{1}(c)$ can then be written as the sum of two contributions

$$
V_{1}(c)=V_{\mathrm{pair}}(c)+n_{\mathrm{cn}}^{1} V_{\mathrm{cn}}(c),
$$

with $V_{\text {pair }}(c)$ the energy difference related to the two atoms of the pair itself given by

$$
\begin{aligned}
V_{\mathrm{pair}}(c)= & \sum_{p=0}^{Z-1} C_{Z-1}^{p} c^{p}(1-c)^{Z-1-p}\left(\left(E_{A}^{p+1}(c)-E_{A}^{p}(c)\right)\right. \\
& \left.-\left(E_{B}^{p+1}(c)-E_{B}^{p}(c)\right)\right)
\end{aligned}
$$

and $V_{\mathrm{cn}}(c)$ is the energy difference related to the $n_{\mathrm{cn}}^{1}$ atoms, which are common neighbors of the pair in the final state

$$
\begin{aligned}
V_{\mathrm{cn}}(c)= & \sum_{p=0}^{Z-2} C_{Z-2}^{p} c^{p}(1-c)^{Z-2-p} \\
& \times\left(c \tilde{E}_{A}^{p+1}(c)+(1-c) \tilde{E}_{B}^{p+1}(c)\right),
\end{aligned}
$$

with $\tilde{E}_{I}^{x}=\left(E_{I}^{x+1}(c)+E_{I}^{x-1}(c)-2 E_{I}^{x}(c)\right) / 2,(1 \leqslant x \leqslant Z-1)$.

We detail now these two contributions

(1) $V_{\text {pair }}(c)$ [cf. Eq. (11)] is a combinatorial sum of the difference in $p$ slope $\Delta_{p}\left[E_{A}^{p}\right]-\Delta_{p}\left[E_{B}^{p}\right]$ with $\Delta_{p}\left[E_{I}^{p}\right]=$ $E_{I}^{p+1}-E_{I}^{p}$

(2) $V_{\mathrm{cn}}(c)$ [cf. Eq. (12)] is a combinatorial sum of the $p$ curvature $\Delta_{p}^{2}\left[E_{I}^{p}\right]=\tilde{E}_{I}^{p}$ of the two constituents.

This means that the ESE model provides an analytical expression of the EPIs taking into account the dependency on both the local environment (related to $p$ ) and the nonlocal environment (related to $c$ ). If the site energies vary linearly with $p$ and are independent of $c$, curvatures are nil, and slopes are constant: $\Delta_{p}\left[E_{A}^{p}\right]=V_{1}^{A A}-V_{1}^{A B}$ and $\Delta_{p}\left[E_{B}^{p}\right]=$ $V_{1}^{A B}-V_{1}^{B B}$. Thus, one recovers the expression of $V_{1}$ in an Ising model: $V_{1}=\left(V_{1}^{A A}+V_{1}^{B B}-2 V_{1}^{A B}\right) / 2$.

In the ESE formalism, the alloy pair interactions in the $R$ th shell of neighbors depends only on the second contributions relative to the $n_{\mathrm{cn}}^{R}$ common neighbors of the pair

$$
V_{R>1}(c)=n_{\mathrm{cn}}^{R} V_{\mathrm{cn}}(c)
$$

For a fcc lattice and with an index $p$ characterizing the local environment restricted to the nearest neighbors, $V_{R>4}(c)=$ 0 and $Z V=\sum_{R} Z_{R} V_{R}=Z V_{\text {pair }}(c)+Z(Z-1) V_{\mathrm{cn}}(c)$ with $Z=12$.

The permutation enthalpy $\Delta H^{\text {perm }}$ is then written as the sum of two contributions

$$
\Delta H^{\mathrm{perm}}=\Delta H_{\mathrm{EPI}}^{\mathrm{perm}}+\Delta H_{\mathrm{coh}, \mathrm{size}}^{\mathrm{perm}},
$$


0

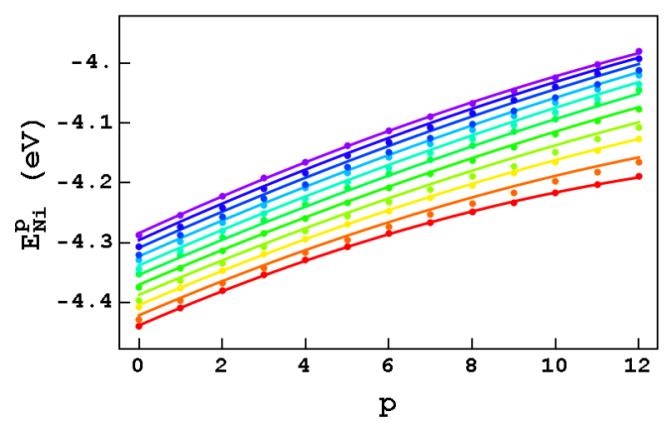

(a)

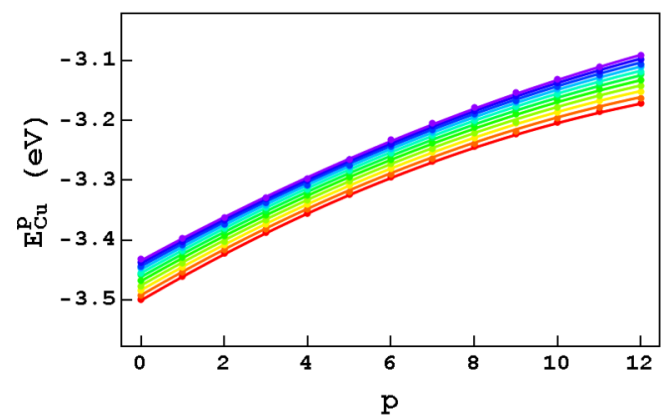

(c)
0.5

1

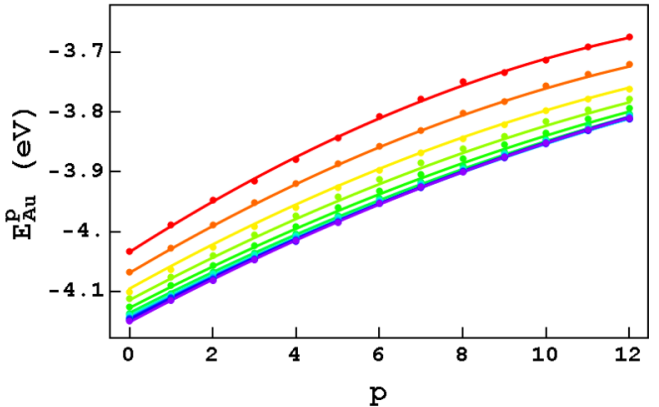

(b)

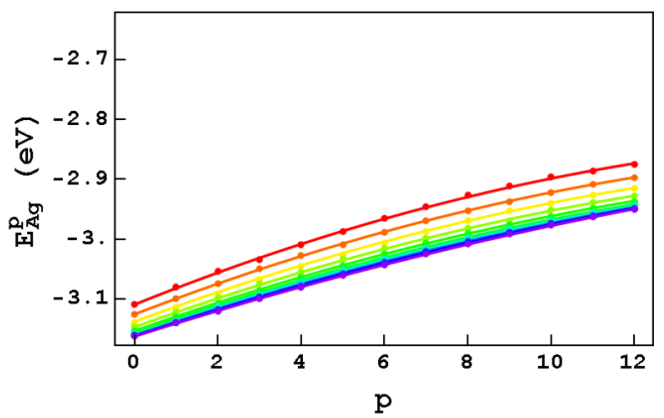

(d)

FIG. 1. Site energy of an atom $I$ as a function of the number $p$ of $A$ neighbors, for different values of the concentration in $\mathrm{A}_{c} \mathrm{~B}_{1-c}$ alloys: (a) $I=\mathrm{Ni}$, (b) $\mathrm{Au}$ for $\mathrm{Au}_{c} \mathrm{Ni}_{1-c}$, (c) $\mathrm{Cu}$, (d) $\mathrm{Ag}$ for $\mathrm{Ag}_{c} \mathrm{Cu}_{1-c}$; with $A=\mathrm{Au}$ in (a) and (b), $\mathrm{Ag}$ in (c) and (d). The color scales between red ( $c=0, B$-pure system) and purple ( $c=1, A$-pure system). The lines are only a guide for the eyes.

with

$$
\begin{aligned}
\Delta H_{\mathrm{EPI}}^{\mathrm{perm}}= & -(1-2 c) Z V(c)-c(1-c) Z \frac{\partial V_{a}(c)}{\partial c}, \\
\Delta H_{\mathrm{coh}, \mathrm{size}}^{\mathrm{perm}}= & Z \tau(c)-c(1-c) Z \frac{\partial V_{b}(c)}{\partial c}+Z \frac{\partial \tau(c)}{\partial c} c \\
& +\frac{\partial E_{B}^{0}(c)}{\partial c} \approx Z \tau(c)+\Delta E_{\Delta c},
\end{aligned}
$$

where $\partial V_{a}(c) / \partial c$ denotes that only the combinatorial sums of the EPIs are differentiated, whereas for $\partial V_{b}(c) / \partial c$, only the site energies are differentiated.

The first contribution $\Delta H_{\mathrm{EPI}}^{\mathrm{perm}}$ is related to the EPIs which characterize the tendency to form homoatomic or heteroatomic bonds. The second contribution $\Delta H_{\text {coh,size }}^{\text {perm }}$ includes $Z \tau(c)$, which is related to the difference in cohesive energies between the two metals (hence the name "coh") and the complementary term [see Eq. (8)] whose variation is related to the derivative of the $E_{I}^{p}(c)$ with respect to $c$ (hence the name "size"). Integration of these two contributions according to Eq. (A12) leads to $\Delta H_{\mathrm{EPI}}^{\text {mixing }}$ and $\Delta H_{\mathrm{coh} \text {,size }}^{\text {mixing }}$

$$
\begin{gathered}
\Delta H_{\mathrm{EPI}}^{\text {mixing }}=-c(1-c) Z V(c), \\
\Delta H_{\mathrm{coh}, \text { size }}^{\text {mixing }}=Z \tau(c) c+E_{B}^{0}(c)-\left[c E_{A}^{Z}(1)+(1-c) E_{B}^{0}(0)\right] .
\end{gathered}
$$

\section{RESULTS}

\section{A. Database of site energies}

In this section, we present the main characteristics of the site energies for $\mathrm{Au}_{c} \mathrm{Ni}_{1-c}$ and $\mathrm{Ag}_{c} \mathrm{Cu}_{1-c}$ rss.

Figure 1 displays the variation of the site energies of $\mathrm{Ni}$ atoms [Fig. 1(a)] and Au atoms [Fig. 1(b)] as a function of the number of $\mathrm{Au}$ nearest neighbors, for a given nominal concentration. As expected, the site energy of a $\mathrm{Ni}$ atom for $p=0$ and $c=0$ is equal to the cohesive energy of $\mathrm{Ni}$, $E_{\mathrm{Ni}}^{0}(0)=-4.44 \mathrm{eV}$ [Fig. 1(a)]. Similarly, the Au cohesive energy is retrieved for $p=12$ and $c=1, E_{\mathrm{Au}}^{12}(1)=-3.81 \mathrm{eV}$ [Fig. 1(b)]. Au being less cohesive than Ni, an atom becomes less cohesive when its local surrounding is richer in $\mathrm{Au}$, i.e., when $p$ increases. In $\mathrm{Ag}_{c} \mathrm{Cu}_{1-c}$, the evolution of the site energies of $\mathrm{Cu}$ atoms [Fig. 1(c)] and $\mathrm{Ag}$ atoms [Fig. 1(d)] with the number of $\mathrm{Ag}$ nearest neighbors is very similar to the one observed for $\mathrm{Ni}$ and $\mathrm{Au}$ in $\mathrm{Au}_{c} \mathrm{Ni}_{1-c}$. Cu being more cohesive than $\operatorname{Ag}\left(E_{\mathrm{Cu}}^{0}(0)=-3.5 \mathrm{eV}\right.$ and $\left.E_{\mathrm{Ag}}^{12}(1)=-2.95 \mathrm{eV}\right), \mathrm{Cu}$ and $\mathrm{Ag}$ atoms become less cohesive when increasing their Ag local environment.

The $c$ dependence of the $E_{I}^{p}(c)$ for a given $p$ value is mainly related to the variation of the lattice parameter of the rss as a function of $c$. As expected, $E_{A}^{12}(c)$ is minimum for $c=1$ [Figs. 1(b) and 1(d)] and $E_{B}^{0}(c)$ for $c=0$ [Figs. 1(a) and 1(c)]. For both alloys, we see that this rule can be extended to the other values of $p: E_{A}^{p}(c)$ is a decreasing function of $c$, whereas $E_{B}^{p}(c)$ is an increasing value of $c$. Note that the variation of the 
site energy with the concentration at a given $p$ value is almost two times higher for the Au-Ni system [Figs. 1(a) and 1(b)] than for the Ag-Cu one [Figs. 1(c) and 1(d)].

Finally, the site energies $E_{I}^{p}(c)$ have two main features that distinguish them from Ising models using EPIs independent of $c$

(1) a nonzero curvature as a function of $p: \partial^{2} E_{I}^{p}(c) / \partial^{2} p \neq$ 0 . This curvature leads to a dependency of the EPIs on the local concentration (given by the value of $p$ ), itself a function of $c$. This local effect is one of the explanations for the asymmetry of the phase diagrams;

(2) a dependency on $c$ at a given $p: \partial E_{I}^{p}(c) / \partial c \neq 0$. As mentioned above, this dependency is related to the variation of the lattice parameter with respect to $c$. When $c$ increases, a dilation occurs from the $\mathrm{Ni}$ lattice parameter to the $\mathrm{Au}$ one in $\mathrm{Au}_{c} \mathrm{Ni}_{1-c}$ and from the $\mathrm{Cu}$ lattice parameter to the $\mathrm{Ag}$ one in $\operatorname{Ag}_{c} \mathrm{Cu}_{1-c}$.

\section{B. Validation of the ESE formalism}

To test the ESE model, we have performed molecular static simulations to get direct values of $\Delta H^{\text {mixing }}$ and $\Delta H^{\text {perm }}$. For a given concentration, the internal energy is averaged over 100 rss. Here, $\Delta H^{\text {mixing }}$ is then deduced from Eq. (A10) and $\Delta H^{\text {perm }}$ from Eq. (A4).

Figures 2(a) and 2(c) show the evolution of the mixing enthalpy as a function of the nominal concentration for both alloys. Here, $\Delta H^{\text {mixing }}$ is positive on the whole range of concentration as expected for alloys with a tendency to phase separate. These results are in good agreement with available experimental data [38], even if $\Delta H^{\text {mixing }}$ is slightly

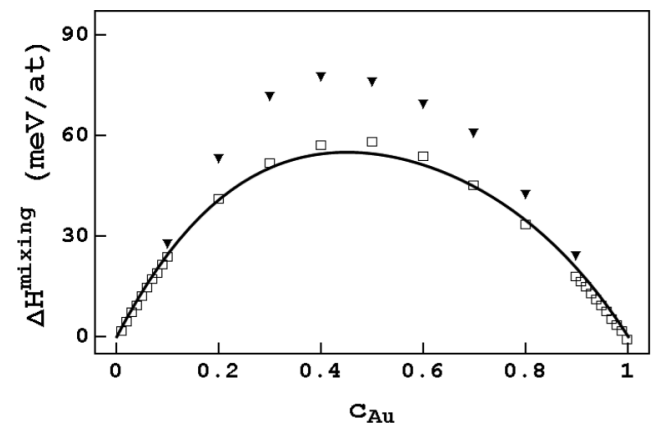

(a)

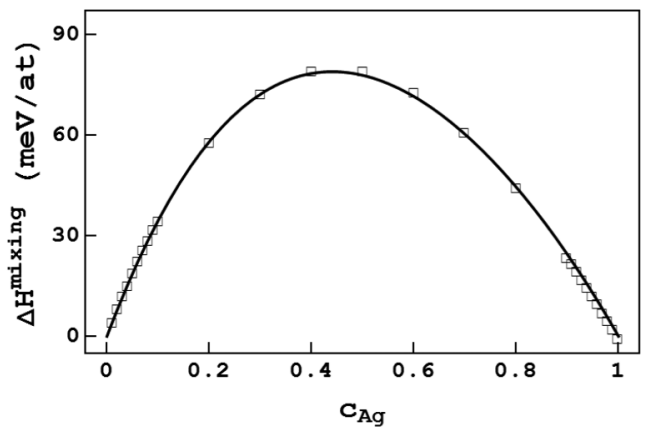

(c) underestimated for Au-Ni rss. Note that a perfect agreement is obtained between the values of $\Delta H^{\text {mixing }}$ predicted by Eq. (3) and the direct values issued from the atomistic simulations. This validates the use of the site energies $E_{I}^{p}(c)$ as long as they can be obtained and the restriction to the nearest-neighbor shell for their dependency on the local environment. If the reconstruction of the ESE-based energy was not correct, it would be necessary to introduce additional local parameters, such as the influence of second neighbors or of some angular terms. Studies on other fcc alloys within the framework of the SMA show that the restriction to the nearest-neighbor shell remains valid to define the local environment for the site energies [63]. On the other hand, a study of the Ni-C system within the fourth-moment approach [64] put in evidence the necessity to integrate the second neighbors in the definition of the local environment [65]. Thus, the pertinence of the local environment definition is a key point of the method, and it must be tested for each system by considering the good reproduction of the mixing enthalpy, for instance.

Similar to $\Delta H^{\text {mixing }}$, a very good agreement is obtained for the permutation enthalpy between the values of $\Delta H^{\text {perm }}$ predicted by Eq. (5) and the direct values issued from the atomistic simulations [see Figs. 2(b) and 2(d)]. For both alloys, $\Delta H^{\text {perm }}$ is a decreasing function of the nominal concentration due to the tendency of these alloys to phase separate.

\section{Comparison between Au-Ni and Ag-Cu: Analysis of differences}

A comparison between both systems indicates that $\Delta H^{\text {mixing }}$ is slightly higher for $\mathrm{Ag}-\mathrm{Cu}$ than for $\mathrm{Au}-\mathrm{Ni}$

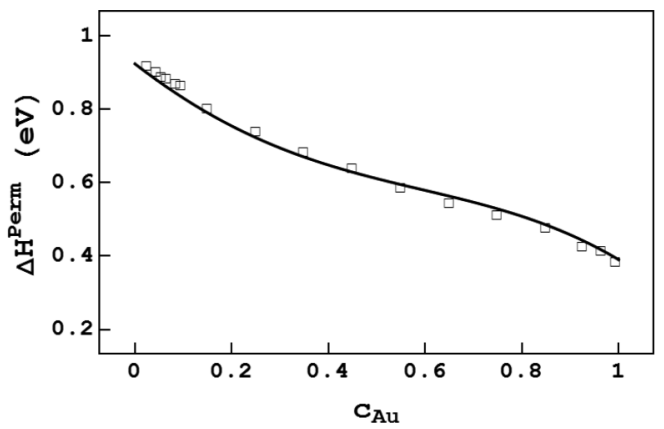

(b)

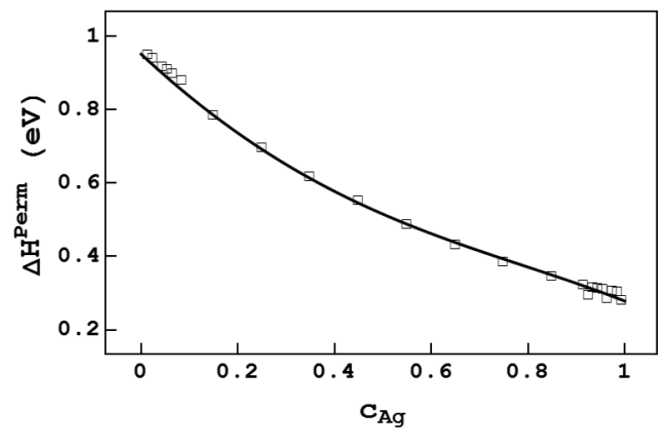

(d)

FIG. 2. Mixing enthalpy $\Delta H^{\text {mixing }}$ as a function of $c$ for (a) $\mathrm{Au}_{c} \mathrm{Ni}_{1-c}$ and (c) $\mathrm{Ag}_{c} \mathrm{Cu}_{1-c}$. Permutation enthalpy $\Delta H^{\text {perm }}$ as a function of $c$ for (b) $\mathrm{Au}_{c} \mathrm{Ni}_{1-c}$ and (d) $\mathrm{Ag}_{c} \mathrm{Cu}_{1-c}$. A comparison between SMA-MC simulations (empty squares) and analytical formulae [Eqs. (3) and (5)] (lines) is also highlighted. In (a) the triangles indicate the experimental data [38]. 


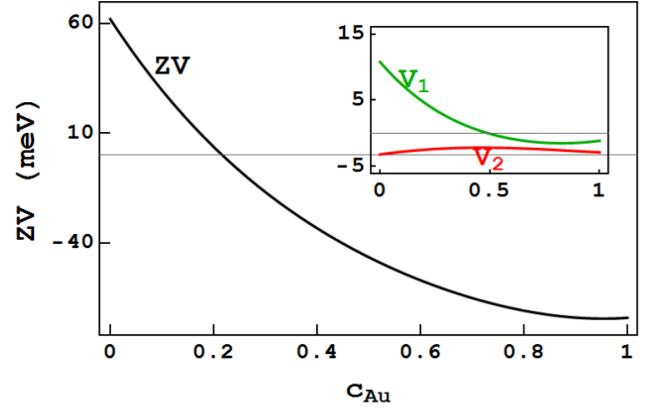

(a)

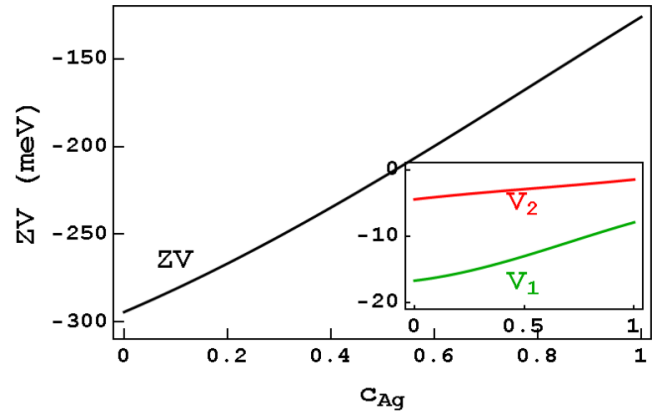

(b)

FIG. 3. Evolution as a function of $c$ of $Z V=\sum_{R=1,4} Z_{R} V_{R}$ (black line), $V_{1}$ (green line), and $V_{2}$ (red line) for (a) $\mathrm{Au}_{c} \mathrm{Ni}_{1-c}$ and (b) $\mathrm{Ag}_{c} \mathrm{Cu}_{1-c}$.

[Figs. 2(a) and 2(c)]. In parallel, the amplitude of variation of $\Delta H^{\text {perm }}$ for $\mathrm{Ag}-\mathrm{Cu}$ is about 1.3 times the $\mathrm{Au}-\mathrm{Ni}$ one [Figs. 2(b) and 2(d)].

As shown in Figs. 2(b) and 2(d), $\Delta H^{\text {perm }}$ is a nonlinear function of $c$, contrary to what is expected in the framework of an Ising model with constant EPIs [see expression in Eq. (A9)]. Within the Ising model, $\Delta H^{\text {perm }}$ varies from $Z \tau-Z V$ for $c \rightarrow 0$ to $Z \tau+Z V$ for $c \rightarrow 1$ with $\Delta H^{\text {perm }}(c=1 / 2)=Z \tau$. We can suspect that a part of the nonlinearity observed for $\Delta H^{\text {perm }}(c)$ is due to a variation of the EPIs with $c$, which is taken into account into the ESE formalism. Figure 3 illustrates the variation of $V_{1}$ [Eq. (10)], $V_{2}$ [Eq. (13)], and $Z V=\sum_{R \leqslant 4} Z_{R} V_{R}$ for the $\mathrm{Au}_{c} \mathrm{Ni}_{1-c}$ alloy [Fig. 3(a)] and for the $\mathrm{Ag}_{c} \mathrm{Cu}_{1-c}$ alloy [Fig. 3(b)]. An unexpected result is the sign reversal observed for $V_{1}$ in Au-Ni solid solutions [Fig. 3(a)]. The positive sign obtained for $c<0.5$ is consistent with the tendency to order at short-range observed experimentally in this system, even if it remains controversial $[20,21,30,37,43,45,66,67]$. Such a sign reversal of $V_{1}$ as a function of $c$ has not been yet reported in the literature, the major part of the studies being focused on alloys near the equiatomic composition. Conversely, for the $\mathrm{Ag}-\mathrm{Cu}$ system, no sign reversal of $V_{1}$ is observed [Fig. 3(b)]. Here, $V_{1}$ remains negative on the whole range of concentration, as it is expected for a system presenting a large miscibility gap. If the behavior of $V_{1}(c)$ strongly differs between both systems, this is not the case for $V_{2}(c)$, which is almost constant and slightly negative for both alloys (Fig. 3). Also, $Z V$ is the quantity that controls the variation of the permutation enthalpy with $c$ in the framework of the Ising model [see Eq. (A9)]. Figure 3

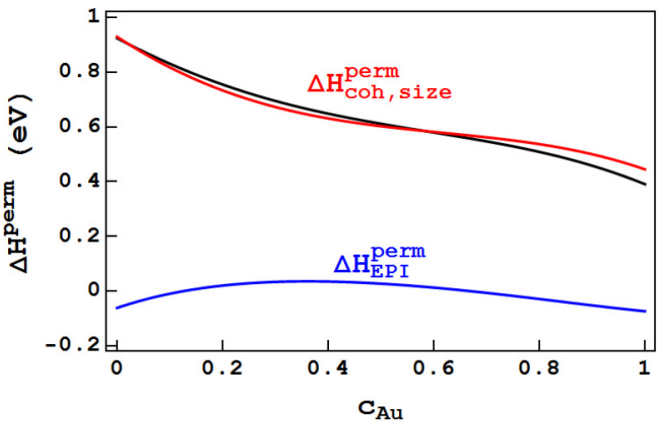

(a)

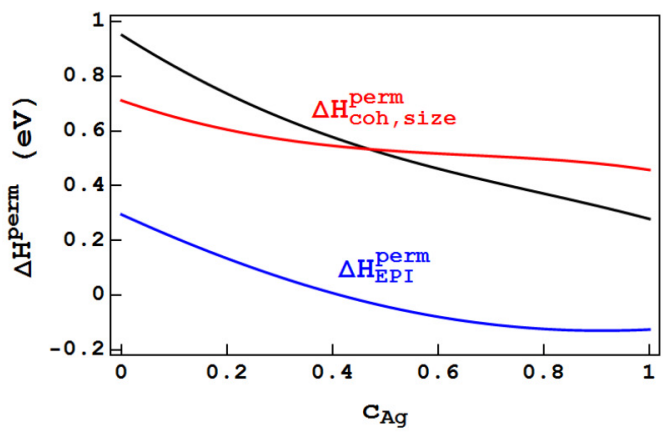

(c)

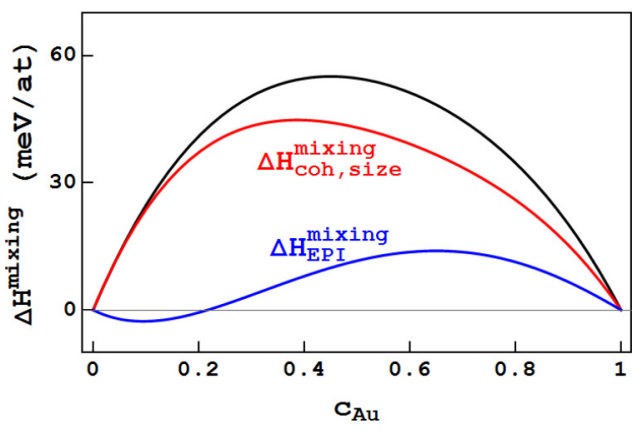

(b)

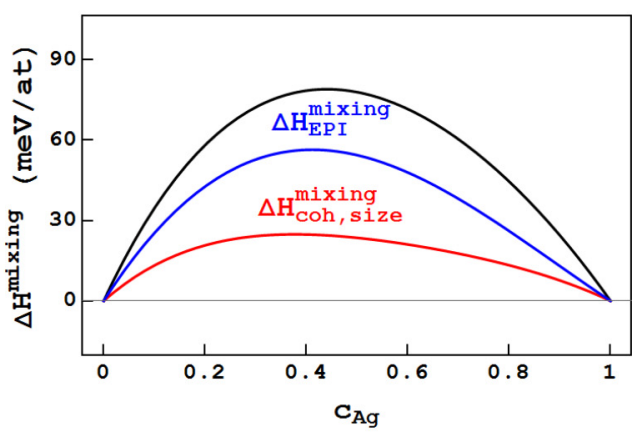

(d)

FIG. 4. Evolution as a function of $c$ of the permutation enthalpy $\Delta H^{\text {perm }}$ (black line) and of its two contributions $\Delta H_{\text {coh, size }}^{\text {perm }}$ (red line) and $\Delta H_{\mathrm{EPI}}^{\text {perm }}$ (blue line) for (a) $\mathrm{Au}_{c} \mathrm{Ni}_{1-c}$ and (c) $\mathrm{Ag}_{c} \mathrm{Cu}_{1-c}$. Evolution as a function of $c$ of the mixing enthalpy $\Delta H^{\text {mixing }}$ (black line) and of its two contributions $\Delta H_{\text {coh, size }}^{\text {mixing }}$ (red line) and $\Delta H_{\mathrm{EPI}}^{\text {mixing }}$ (blue line) for (b) $\mathrm{Au}_{c} \mathrm{Ni}_{1-c}$ and (d) $\mathrm{Ag}_{c} \mathrm{Cu}_{1-c}$. 


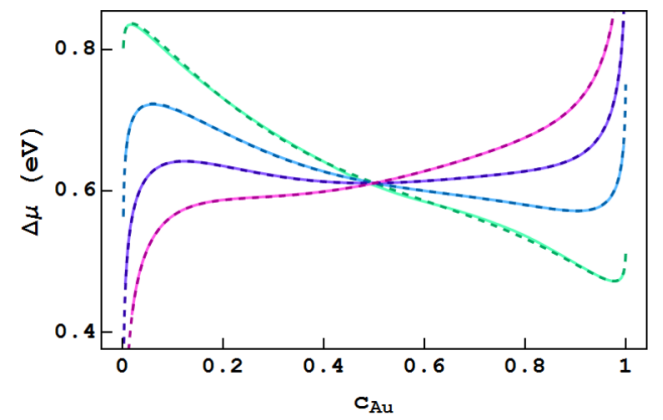

(a)

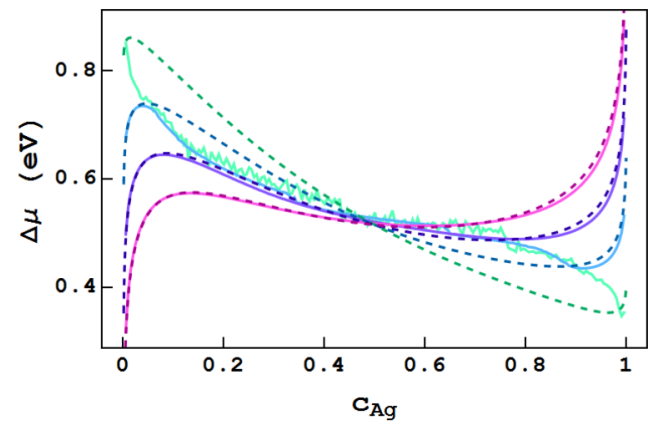

(b)

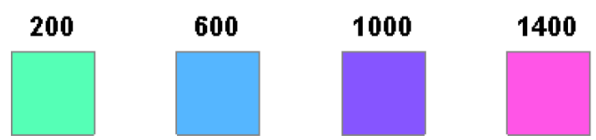

FIG. 5. Comparison between ESE-MC (lines) and ESE-MFA (dashed lines) isotherms $\Delta \mu(c)$ at different temperatures for $(a) \mathrm{Au}_{c} \mathrm{Ni}_{1-c}$ and (b) $\mathrm{Ag}_{c} \mathrm{Cu}_{1-c}$. The color scales between green $(T=200 \mathrm{~K})$ and pink $(T=1400 \mathrm{~K})$ by increments of $400 \mathrm{~K}$.

shows that the behavior of $Z V$ is dominated by the behavior of $V_{1}$. If the positive sign of $\Delta H^{\text {mixing }}$ and the negative slope of $\Delta H^{\text {perm }}$ are consistent with the negative value of $Z V$ for $\mathrm{Ag}-\mathrm{Cu}$, this is not the case for $\mathrm{Au}-\mathrm{Ni}$, at least within the Ising model. These observations call for the following questions: can we explain the Au-Ni behavior? What is the origin of the differences observed between the Ag- $\mathrm{Cu}$ and $\mathrm{Au}-\mathrm{Ni}$ systems?

To address these questions, we detail the two contributions of both the permutation enthalpy [see Eqs. (14)-(16)] and the mixing enthalpy [Eqs. (17)-(18)] for Au-Ni [Figs. 4(a) and 4(b)] and for Ag-Cu [Figs. 4(c) and 4(d)]. Firstly, $\Delta H^{\text {perm }}$ [Eq. (5)] and $\Delta H^{\text {mixing }}[$ Eq. (3)] are well reproduced by the sum of the two contributions. Secondly, $\Delta H_{\mathrm{EPI}}^{\mathrm{perm}}$ is very small for Au-Ni when compared to $\Delta H_{\text {coh,size }}^{\text {perm }}$ [Fig. 4(a)], whereas it is not the case for Ag-Cu [Fig. 4(c)].

It is important here to realize that SRO parameter is driven by the EPIs and thus by $\Delta H_{\mathrm{EPI}}^{\mathrm{perm}}$ [Eq. (15)], whereas the phase diagram is controlled by the variation of the permutation enthalpy as a function of $c$. This variation is mainly due to $\Delta H_{\mathrm{coh} \text {,size }}^{\text {perm }}[\mathrm{Eq} .(16)]$ for $\mathrm{Au}-\mathrm{Ni}$, although the variation of $\Delta H_{\mathrm{EPI}}^{\mathrm{perm}}$ is not completely negligible. Conversely, the variation of $\Delta H_{\mathrm{EPI}}^{\text {perm }}$ as a function of $c$ is the leading term for $\mathrm{Ag}-\mathrm{Cu}$. Note that a more detailed analysis shows that, for both systems, $\Delta H_{\text {coh,size }}^{\text {perm }}$ and its variation as a function of $c$ is largely dominated by the cohesive contribution $Z \tau(c)$.

Thus, the decomposition of $\Delta H^{\text {perm }}$ allows one to predict

(1) for Au-Ni alloys: a weak SRO (with a reversal between ordering and phase separating tendency as a function of $c$ ) due to the small value of $\Delta H_{\mathrm{EPI}}^{\mathrm{perm}}$ and a phase diagram driven by the slope of $\Delta H^{\text {perm }}(c)$, itself dominated by the slope of $\Delta H_{\text {coh,size }}^{\text {perm }}(c)$ and more precisely by the slope of $Z \tau(c)$. Thus, SRO and phase diagram are not driven by the same effect;

(2) for Ag-Cu alloys: a strong SRO (with a phase separating tendency on the whole range of $c$ ) due to the high value of $\Delta H_{\mathrm{EPI}}^{\mathrm{perm}}$ and a phase diagram driven by the slope of $\Delta H^{\text {perm }}(c)$, itself dominated by the slope of $\Delta H_{\mathrm{EPI}}^{\mathrm{perm}}(c)$. For this system, SRO and phase diagram are mainly driven by the same effect, i.e., $\Delta H_{\mathrm{EPI}}^{\mathrm{perm}}$.

These features can directly be transposed to the relative weights of $\Delta H_{\mathrm{EPI}}^{\text {mixing }}\left[\mathrm{Eq}\right.$. (17)] and $\Delta H_{\text {coh,size }}^{\text {mixing }}[$ Eq. (18)] in
$\Delta H^{\text {mixing }}\left[\right.$ Eq. (3)] both for $\mathrm{Au}-\mathrm{Ni}$ and $\mathrm{Ag}-\mathrm{Cu}: \Delta H_{\mathrm{coh} \text {,size }}^{\text {mixing }}$ is predominant for $\mathrm{Au}-\mathrm{Ni}$, whereas $\Delta H_{\mathrm{EPI}}^{\text {mixing }}$ is the main contribution for $\mathrm{Ag}-\mathrm{Cu}$.

\section{ESE isotherms $\Delta \mu(c)$}

To obtain the phase diagrams, we calculate the isotherms $\Delta \mu(c)$ with rigid-lattice ESE-MC simulations in the canonical ensemble. We recall that, in this ensemble, the nominal concentration $c$ and the temperature $T$ are fixed, the difference in chemical potentials $\Delta \mu$ being determined via Widom's method [62]. Here, the ESE-MC isotherms are compared with the ESE-MFA ones.

For $\mathrm{Au}-\mathrm{Ni}$, isotherms $\Delta \mu(c)$ are monotonic increasing functions at high temperatures $[T=1400 \mathrm{~K}$, Fig. 5(a)]. As the temperature decreases, the slope of the isotherm decreases, becomes null, and isotherms are no longer monotonic at low temperatures $[T<1000 \mathrm{~K}$, Fig. 5(a)]. The presence of a decreasing part is the signature of a phase separation, and we can use the rule of equal areas to obtain the solubility limits. This rule ensures that the two phases, one rich in $\mathrm{Au}$ and the other one rich in $\mathrm{Ni}$, have the same free energy, each phase being at its own equilibrium parameter since the energetic quantities of the ESE model depends on the lattice parameter via the nominal concentration. Hence, rigidlattice ESE-MC simulations in the canonical ensemble lead to the incoherent phase diagram as for a real alloy, while only coherent configurations are sampled. This is also supported by the analysis of the local chemical order, which is quantified by the Warren-Cowley SRO parameter in the first neighboring shell, $\alpha_{110}(c, T)=1-P_{110}^{A B}(c, T) / c$, where $P_{110}^{A B}(c, T)$ is the probability to have heteroatomic bonds in the first shell [48]. A rss is characterized by $\alpha_{110}=0$. For an ordered configuration $\alpha_{110}<0$, whereas $\alpha_{110}>0$ indicates a tendency to form homoatomic bonds. Figure 6(a) depicts the SRO evolution as a function of the composition at different temperatures for $\mathrm{Au}$ Ni. One can observe a sign change at $c \approx 0.5$ [Fig. 6(a)]. The first regime displays a slight tendency to form heteroatomic bonds $(c<0.5)$, while the second regime indicates a tendency to form homoatomic bonds $(c>0.5)$. This sign change is fully 


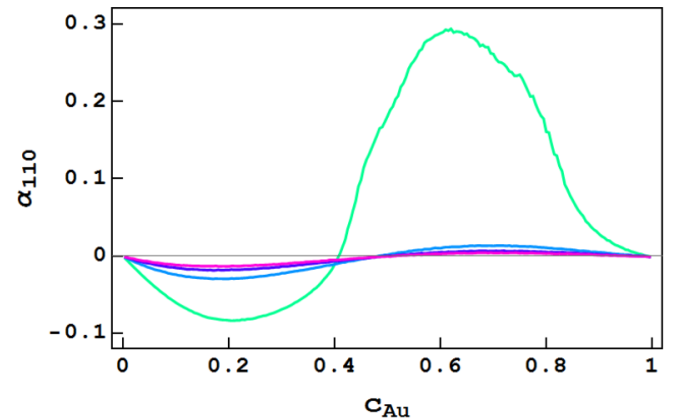

(a)

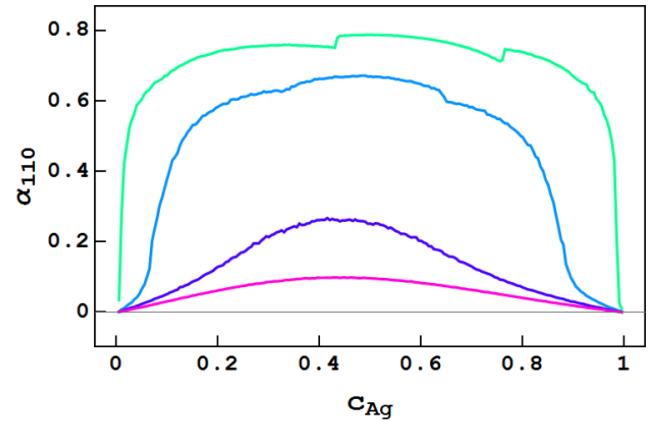

(b)

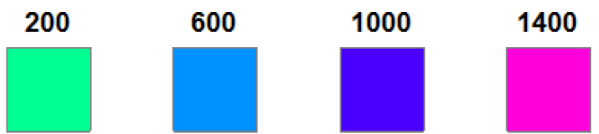

FIG. 6. Short-range order parameter $\alpha_{110}$ as a function of $c$ issued from ESE-MC simulations at different temperatures for (a) Au $\mathrm{Ni}_{1-c}$ and (b) $\operatorname{Ag}_{c} \mathrm{Cu}_{1-c}$. The color scales between green $(T=200 \mathrm{~K})$ and pink $(T=1400 \mathrm{~K})$ by increments of $400 \mathrm{~K}$. Note that the scale of the $\mathrm{Y}$ axis for $\mathrm{Au}-\mathrm{Ni}$ is smaller by approximately a factor of 2 than the one for $\mathrm{Ag}-\mathrm{Cu}$.

correlated with the sign reversal observed for $V_{1}(c)$ [Fig. 3(a)]. The very small value of $\alpha_{110}$, even at low temperatures [see Fig. 6(a)], indicates that no coherent phase separation occurs and that isotherms are of van der Waals type [Fig. 5(a)], which explains also the perfect agreement observed between the ESE-MFA and the ESE-MC isotherms. Moreover, $\Delta \mu$ being close to $\Delta H^{\text {perm }}$ on the whole range of nominal concentration, it shows that the main driving force that controls the phase separation is the variation of $\Delta H_{\text {coh,size }}^{\text {perm }} \approx Z \tau(c)$ as a function of $c$ and not the EPI's contribution. All these results allow one to establish the relative stability between all possible equilibrium states, from the most to the less stable one: incoherent phase separation/rss/coherent phase separation. At low temperatures, ordered phases are expected to occur (in relative stability) between incoherent phase separation and the rss.

For $\mathrm{Ag}-\mathrm{Cu}$, isotherms always display a decreasing part, but two regimes in temperature can still be distinguished [Fig. 5(b)]: At low temperatures, the slope of the isotherm near $c \approx 0.5$ does not depend on the temperature, whereas at high temperatures, it varies with the temperature. In the first regime (low $T$ ), the high positive values of the SRO parameter characterizes a two-phase state [Fig. 6(b)]. The strong tendency to favor homoatomic bonds at low temperatures
[Fig. 6(b)] is the signature of a coherent phase separation. It is in good agreement with the fact that $\Delta H_{\mathrm{EPI}}^{\mathrm{perm}}$ largely controls the variation of $\Delta H^{\text {perm }}$ as a function of $c$. Hence, the loops of the isotherms correspond to the nucleation and growth of a second phase with the same lattice parameter from the parent phase and differ from the van der Waals loops of the ESE-MFA isotherms [Fig. 5(b)]. In the second regime (high $T$ ), the weak positive values of the SRO parameter [Fig. 6(b)] indicates that configurations are close to rss, and isotherms tend towards van der Waals loops, as shown by the perfect match with the ESE-MFA isotherms [Fig. 6(b)]. The effect of $\Delta H_{\mathrm{coh}, \text { size }}^{\mathrm{perm}}$ being not negligible, it contributes to an increase of the incoherent critical temperature and thus to the existence of a temperature range below $T_{c}$ where local order is very weak. Hence, this type of isotherm indicates the following relative stability between all possible equilibrium states: incoherent phase separation/coherent phase separation/rss at low temperatures, and then incoherent phase separation/rss/coherent phase separation at high temperatures, but still below $T_{c}$.

\section{E. Phase diagrams}

In this section, we compare the incoherent experimental phase diagrams [68] with those obtained with ESE-MC

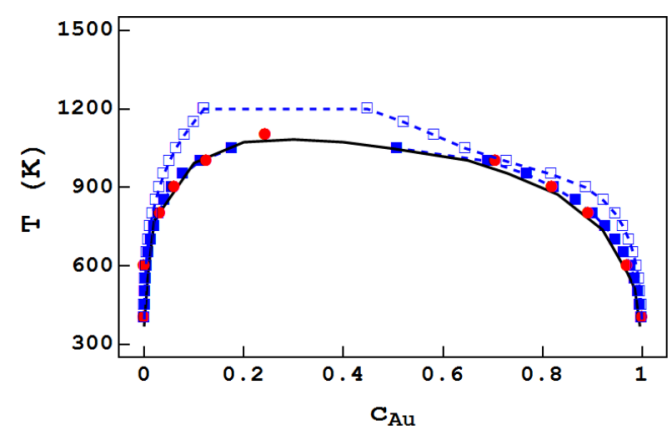

(a)

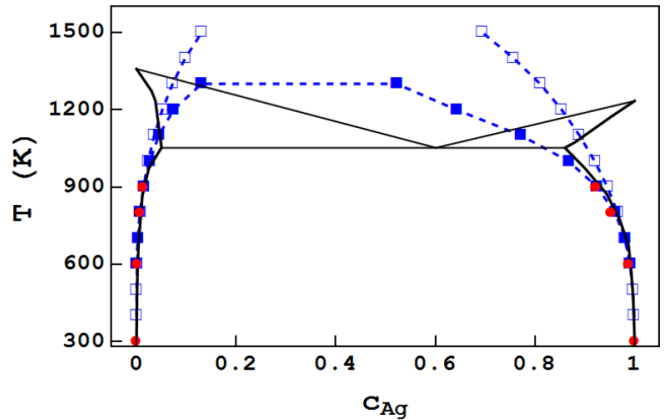

(b)

FIG. 7. Experimental (black line) and calculated phase diagram (symbols) of (a) $\mathrm{Au}_{c} \mathrm{Ni}_{1-c}$ and (b) $\mathrm{Ag}_{c} \mathrm{Cu}_{1-c}$. Phase diagrams are issued from SMA-MC simulations (red points), ESE-MC simulations including $\Delta S_{\text {vib }}^{\text {perm }}$ (filled blue squares), ESE-MC simulations without $\Delta S_{\text {vib }}^{\text {perm }}$ (empty blue squares). 
simulations, including or not the contribution of the vibrational part of the permutation entropy $\Delta S_{\mathrm{vib}}^{\text {perm }}$ (see Appendix B) and SMA-MC simulations in the canonical ensemble for both alloys (Fig. 7). To take into account $\Delta S_{\text {vib }}^{\text {perm }}$ in the ESE-MC simulations, Widom's procedure, which allows the determination of $\Delta \mu$, is modified as follows: the energy change associated with the random insertion of a test particle $\Delta H^{\text {perm }}$ is replaced by $\Delta H^{\text {perm }}-T \Delta S_{\text {vib }}^{\text {perm }}$.

For both alloys, Fig. 7 shows that the asymmetry of the phase diagram issued from ESE-MC simulations is in agreement with the experimental one, but the miscibility gap is too large, and the critical temperature is overestimated. Adding $\Delta S_{\text {vib }}^{\text {perm }}$ in the ESE-MC simulations leads to an increase of the solubility limits and to a decrease of $T_{c}$ (see Fig. 7), in agreement with the results obtained by Asta and Foiles with their SOE-EAM formalism [30]. Here, $\Delta S_{\text {vib }}^{\text {mixing }}$ being positive and greater for $\mathrm{Ag}-\mathrm{Cu}$ than for $\mathrm{Au}-\mathrm{Ni}$, its effect is more significant for Ag-Cu alloys. Thus, one gets a good agreement between the phase diagrams calculated on an effective lattice (ESE-MC including the vibrational entropy contribution) and those issued from off-lattice SMA-MC simulations. This shows that the ESE can be used for the determination of phase diagrams from rigid-lattice simulations as long as they can be determined.

For both alloys, phase diagrams predict an incoherent phase separation but do not provide any clue on the differences in behavior between the two systems. For Au-Ni [Fig. 7(a)], the incoherent phase separation is mainly driven by the elastic effect, while the SRO is related to the EPIs and undergoes a sign change as a function of the nominal concentration. Thus, a tendency to order is observed for $c \lesssim 0.5\left(\alpha_{110}<0\right.$ and $V_{1}>0$ ) for an alloy having a large miscibility gap. Moreover, the expected tendency to phase separate is recovered for $c \lesssim 0.5\left(\alpha_{110}>0\right.$ and $\left.V_{1}<0\right)$, but with low values of the SRO parameter and thus with configurations close to rss, as previously mentioned. Hence, the coherent phase diagram, driven by the EPIs but hidden by the incoherent phase diagram, predicts an ordering transition for $c \lesssim 0.5$ and a phase separation transition for $c \gtrsim 0.5$.

For $\mathrm{Ag}-\mathrm{Cu}$, an incoherent phase separation occurs at all temperatures up to the eutectic temperature [Fig. 7(b)], but a coherent phase separation may also occur at low temperatures when the values of the SRO parameter are high $\left(\alpha_{110} \rightarrow 1\right)$. Disordered configurations are observed in MC simulations at higher temperatures $\left(\alpha_{110} \rightarrow 0\right)$ due to the elastic contribution, which is lower than the EPI's but significant. Thus, as for $\mathrm{Au}-\mathrm{Ni}$, the coherent phase diagram is also hidden by the incoherent one.

ESE-MC and SMA-MC phase diagrams are in good agreement for both alloys, the overestimation of $T_{c}$ being about $50 \mathrm{~K}$ for Au-Ni. Moreover, note that since SRO is weak (because the contribution of $\Delta H_{\text {coh,size }}^{\text {perm }}$ is either dominant or significant), the ESE-MFA reproduces well the results issued from ESE-MC simulations, leading to a fairly accurate estimate of both the critical temperature and the solubility limits, despite the use of the MFA.

\section{DISCUSSION AND CONCLUSION}

\section{A. ESE model}

In this paper, we propose a new effective energetic model to describe $A_{c} B_{1-c}$ binary alloys. It is based on the determination of site energies of rss, taking into account relaxation of the atomic positions and volume optimization. Site energies depend both on the local surrounding of the considered site via the number of its $A$ nearest neighbors and on the nominal concentration. The ESE model can be implemented in rigid-lattice $\mathrm{MC}$ simulations and in mean-field modeling. In this paper, the site energies have been calculated using SMA interatomic potentials, but in the future it should also be possible to carry out NRL-TB or ab initio calculations.

The ESE approach provides analytical formulae of both the permutation enthalpy and the mixing enthalpy on the whole range of nominal concentration. Moreover, the EPIs can be calculated in the entire concentration range. The mapping of this formalism with a generalized Ising model allows for the identification of two driving forces that control the phase diagrams, namely the EPI's effect that governs the local chemical order and the "coh-size" effect, which is related to the variation of the lattice parameter with the nominal concentration. Many attempts have been done to separate the chemical and the size effects, each separation depending on the chosen reference state. The strength of the ESE model is that it does not depend on virtual unrelaxed configurations; all energies in the reference state are relaxed and depend on both the local chemical environment and the nominal concentration. The proposed method is very general in its principle. It can be applied to substitutional or interstitial alloys, to perfect crystals as well as defects (grain boundaries, surfaces). As an example, its extension to superficial segregation requires the calculation of the site energies $E_{I, n}^{p}\left(c_{m}\right)$ on all type of sites $n$ as a function of the local concentrations $c_{m}$, where $n$ and $m$ represent the type of site index (for example the $n$th or $m$ th plane parallel to the surface). The main idea is to determine how the relevant quantities (permutation and mixing enthalpies) depend on the local concentrations near the surface and to detail the interplay of the chemical and size effects.

When phase separation can be described within a simple Ising model, the critical temperature issued from the MFA deviates from the exact one. On the contrary, for phase separation controlled by the coh-size contribution, namely by the nominal concentration, the MFA is expected to be almost exact. The relative weight of the two contributions being strongly different from one to the other system, very different overestimation of the critical temperature is expected. The higher the weight of the coh-size contribution, the more exact the critical temperature.

Applying a MFA treatment to canonical SMA isotherms in the range of temperature where local order is weak allows us to evaluate the vibrational entropy of permutation on the whole concentration range. This vibrational contribution can then be included in the ESE modeling. Taking into account $\Delta S_{\mathrm{vib}}^{\text {mixing }}$ leads to a decrease of the critical temperature and an increase of the solubility limits. Our results confirm that the contribution of the vibrational entropy plays an important role in the case of $\mathrm{Cu}-\mathrm{Ag}$ [49-51].

\section{B. Size-mismatched alloys}

The matching between a generalized Ising model and the ESE model has been applied to two alloys that present a large miscibility gap and a large difference in atomic size. 
For these alloys, the interplay between the displacive disorder and chemical contributions is difficult to determine.

The identification of the chemical contribution and of the coh-size contribution allows for the quantification of their relative weight. We have shown that the coh-size contribution of the mixing enthalpy is quite similar for both alloys, while the chemical contribution differs strongly. This difference is mainly due to the nearest-neighbor EPI $V_{1}$. For Ag-Cu, $V_{1}$ is negative in the entire concentration range, whereas it is positive for $\mathrm{Ni}(\mathrm{Au})$ and negative for $\mathrm{Au}(\mathrm{Ni})$ solid solutions. Diffuse scattering experiments could be done to check this result. Actually, it is not possible to prove the existence of such a difference in behavior from the knowledge of either the phase diagrams or the dissolution enthalpies. We hope that this paper will give rise to further experimental studies devoted to the determination of $V_{1}$, not only for a few values of the concentration but on the whole concentration range.

The evolution of $V_{1}$ as a function of $c$ has an impact on the local chemical order. Hence, we suggest that SRO should be analyzed in the entire concentration range. When the elastic contribution is significant for systems with large size mismatch, the evolution of SRO with temperature gives information on the difference between the incoherent and the coherent critical temperatures.

Finally, we have established the relative stabilities between the possible equilibrium states for both alloys. One can wonder how these hierarchies evolve with the size mismatch. Determination of maps according to the chemical and the size effects is under progress to assess this point.

\section{ACKNOWLEDGMENT}

The authors wish to thank C. Mottet, G. Tréglia, and H. Amara for very fruitful discussions.

\section{APPENDIX A: PERMUTATION ENTHALPY AND ISING MODEL}

Matching the ESE model and the SMA-MC results with MFA allows a detailed analysis of the main thermodynamic driving forces. We recall in this appendix the characteristics of an Ising model developed in the MFA. The $A_{c} B_{1-c}$ system is described by the Hamiltonian [69]

$$
H=\frac{1}{2} \sum_{I J} \sum_{n, m \neq n} p_{n}^{I} p_{m}^{J} V_{n m}^{I J},
$$

where $p_{n}^{I}$ is the occupation number equal to 1 if the site $n$ is occupied by an $I$ atom $(I=A, B)$, and 0 otherwise. Here, $V_{n m}^{I J}$ is the pair interaction between an atom $I$ at site $n$ and an atom $J$ at site $m$.

Using the Bragg-Williams approximation, the energy of the system can be expressed in terms of the energetic parameters of the Ising model [8]

$$
\langle H\rangle / N_{\mathrm{at}}=\sum_{R} Z_{R} \tau_{R} c-c(1-c) \sum_{R} Z_{R} V_{R}+\sum_{R} Z_{R} V_{R}^{B B} / 2,
$$

with $V_{R}=\left(V_{R}^{A A}+V_{R}^{B B}-2 V_{R}^{A B}\right) / 2$ the alloy pair interactions which characterize the tendency to form homoatomic
$\left(V_{R}<0\right)$ or heteroatomic $\left(V_{R}>0\right)$ bonding between $R$ th neighbors, $\tau_{R}=\left(V_{R}^{A A}-V_{R}^{B B}\right) / 2$, and $Z_{R}$ the coordination number for the $R$ th shell of neighbors. Here, $\sum_{R} Z_{R} \tau_{R}$ is equal to the difference in cohesive energies between pure metals $A$ $\left(E_{\mathrm{coh}}^{A}\right)$ and $B\left(E_{\mathrm{coh}}^{B}\right)$. For the sake of simplicity, we omit the index of the shell of neighbors in the following equations, and we denote $Z V$ instead of $\sum_{R} Z_{R} V_{R}$ and $Z \tau$ instead of $\sum_{R} Z_{R} \tau_{R}$

The minimization of the grand-canonical Gibbs free energy with respect to the nominal concentration $c$ leads to the following relationship:

$$
\Delta \mu=\frac{1}{N_{\mathrm{at}}}\left(\frac{\partial\langle H\rangle}{\partial c}-T \frac{\partial S}{\partial c}\right),
$$

with $\Delta \mu=\mu_{A}-\mu_{B}$ the difference in chemical potentials between $A$ and $B$ [59,60], $T$ the temperature, and $S$ the entropy. In Eq. (A3), the first term of the right-hand side corresponds to the permutation enthalpy, i.e., the enthalpy change of the system when a $B$ atom is switched in an $A$ atom

$$
\Delta H^{\text {perm }}=\frac{\partial\langle H\rangle}{\partial N_{A}}=\frac{1}{N_{\mathrm{at}}} \frac{\partial\langle H\rangle}{\partial c} .
$$

The second term is related to the permutation entropy $\Delta S^{\text {perm }}=\partial S / \partial N_{A}=\partial S /\left(N_{\text {at }} \partial c\right)$. These definitions being available whatever the statistical approximation, Eqs. (A3) and (A4) provide the general equation

$$
\Delta \mu=\Delta H^{\text {perm }}-T \Delta S^{\text {perm }} .
$$

On a rigid lattice, the vibrational part of the permutation entropy $\Delta S_{\text {vib }}^{\text {perm }}$ is neglected. In the MFA, the configurational entropy is given by [70]

$$
S^{\text {config }}=-N_{\text {at }} k_{B}(c \ln c+(1-c) \ln (1-c)) .
$$

Thus, the configurational entropy of permutation is

$$
\Delta S_{\text {config }}^{\text {perm }}=-k_{B} \ln \frac{c}{1-c} .
$$

Combining Eqs. (A5) and (A7) leads to the expression of isotherm within MFA [8]

$$
\frac{c}{1-c}=\exp \left(-\frac{\Delta H^{\text {perm }}-\Delta \mu}{k_{B} T}\right) .
$$

The chemical equilibrium configuration is thus controlled by the permutation enthalpy. It can be deduced from Eqs. (A2) and (A4). In the case of constant energetic parameters ( $\tau$ and $V$ ), the derivation yields [8]

$$
\Delta H^{\text {perm }}=Z \tau-(1-2 c) Z V .
$$

Note that the permutation enthalpy is related to the mixing enthalpy. Indeed, the mixing enthalpy being

$$
\Delta H^{\text {mixing }}=\langle H\rangle / N_{\mathrm{at}}-\left(c E_{\mathrm{coh}}^{A}+(1-c) E_{\mathrm{coh}}^{B}\right),
$$

Eqs. (A4) and (A10) lead to the following relationship between the permutation enthalpy and the mixing enthalpy:

$$
\Delta H^{\text {perm }}=\frac{\partial \Delta H^{\text {mixing }}}{\partial c}+\left(E_{\mathrm{coh}}^{A}-E_{\mathrm{coh}}^{B}\right) .
$$

Conversely, integration of the permutation enthalpy on the whole range of nominal concentration provides the mixing 


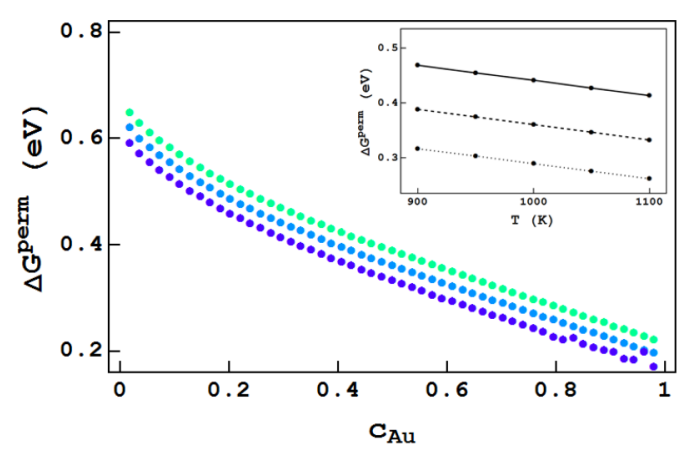

(a)

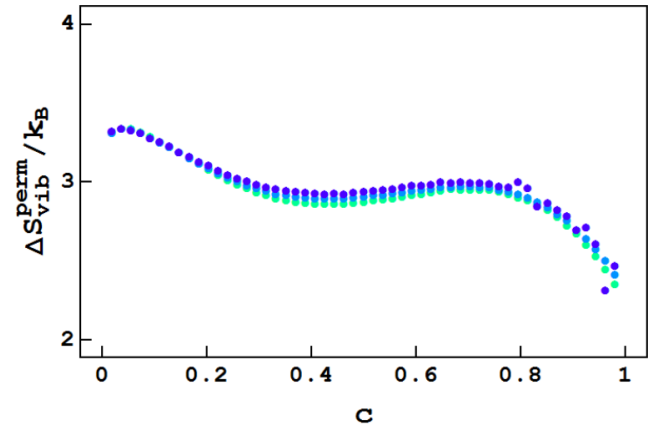

(b)

FIG. 8. Evolution as a function of $c$ of (a) the free permutation enthalpy $\Delta G^{\text {perm }}$ and (b) the vibrational entropy of permutation $\Delta S_{\text {vib }}^{\text {perm }}$ issued from SMA-MC simulations at different temperatures for $\mathrm{Au}_{c} \mathrm{Ni}_{1-c}$. (a) $T=900 \mathrm{~K}$ : green, $T=1000 \mathrm{~K}$ : blue, and $T=1100 \mathrm{~K}$ : dark blue. Inset in (a) shows the evolution of $\Delta G^{\mathrm{perm}}(c)$ as a function of $T$ for $c=0.3$ (line), $c=0.5$ (dashed line), and $c=0.7$ (dotted line). (b) The colors indicate three different pairs of temperatures in the range of 900-1100 K.

enthalpy

$$
\Delta H^{\text {mixing }}(c)=\int_{0}^{c} \Delta H^{\mathrm{perm}}(u) d u-c\left(E_{\mathrm{coh}}^{A}-E_{\mathrm{coh}}^{B}\right) .
$$

For alloys with a tendency to phase separate $(V<0)$, the permutation enthalpy decreases with increasing nominal concentration [Eq. (A9)]. This leads to the existence of a decreasing part in the bulk isotherm $\Delta \mu(c)$ [see Eq. (A8)] at temperatures lower than a critical temperature $T_{c}$, the temperature above which the decreasing part of the isotherms disappears. In MFA, the critical temperature is given by $T_{c}^{\mathrm{MFA}}=-Z V / 2 k_{B}$ [69]. Then at temperatures higher than $T_{c}, \Delta \mu$ becomes a monotonic increasing function of $c$. The determination of the solubility limits at different temperatures can be obtained either from the isotherms using the equal areas rule or from the mixing free energy using the common-tangent rule. However, MFA usually overestimates the value of the critical temperature and the width of the miscibility gap. For example, in the case of the fcc lattice with pair interactions restricted to nearest neighbors $T_{c}^{\mathrm{MFA}} / T_{c}=1.22$ [71].

As previously mentioned, Eq. (A9) is obtained assuming that the energetic parameters do not depend on the nominal concentration. Taking into account such a dependency, the expression of $\Delta H^{\text {perm }}$ becomes

$$
\begin{aligned}
\Delta H^{\mathrm{perm}}= & Z \tau(c)-(1-2 c) Z V(c)-c(1-c) \frac{\partial Z V(c)}{\partial c} \\
& +\frac{\partial Z \tau(c)}{\partial c} c+\frac{1}{2} \frac{\partial Z V^{B B}(c)}{\partial c} .
\end{aligned}
$$

Equation (A13) extends the Eq. (A9) by summing up the variation of the energetic parameters due to the change of nominal concentration when a permutation $B \rightarrow A$ occurs.

Finally, theoretical and experimental studies have established that the contribution of the permutation vibrational entropy can be significant $[15,21,22,28-30,43,49-51]$. Therefore, Eq. (A8) is extended to take into account the vibrational entropy of permutation $\Delta S_{\mathrm{vib}}^{\mathrm{perm}}$

$$
\frac{c}{1-c}=\exp \left(-\frac{\Delta H^{\mathrm{perm}}-\mathrm{T} \Delta S_{\mathrm{vib}}^{\mathrm{perm}}-\Delta \mu}{k_{B} T}\right),
$$

where $\Delta S_{\mathrm{vib}}^{\mathrm{perm}}$ corresponds to the change in the vibrational part of the permutation entropy when a solvent atom is turned in a solute atom. Moreover, we can reasonably consider that $\Delta S_{\mathrm{vib}}^{\text {perm }}$ and $\Delta H^{\text {perm }}$ are independent of temperature, at least for temperatures lower than the fusion temperature.

The vibrational part of the mixing entropy is deduced from $\Delta S_{\text {vib }}^{\text {perm }}$ following

$$
\Delta S_{\mathrm{vib}}^{\text {mixing }}(c)=\int_{0}^{c} \Delta S_{\mathrm{vib}}^{\mathrm{perm}}(u) d u-c\left(S_{\mathrm{vib}}^{A}-S_{\mathrm{vib}}^{B}\right),
$$

with $S_{\text {vib }}^{A}$ and $S_{\text {vib }}^{B}$ being the vibrational entropies of pure metals.

\section{APPENDIX B: THE PERMUTATION VIBRATIONAL ENTROPY}

To account for the vibrational entropy of permutation into the ESE simulations, we analyze the SMA-MC isotherms via the mean-field approach. In canonical SMA-MC simulations, exchanges between two atoms $A$ and $B$, small displacements

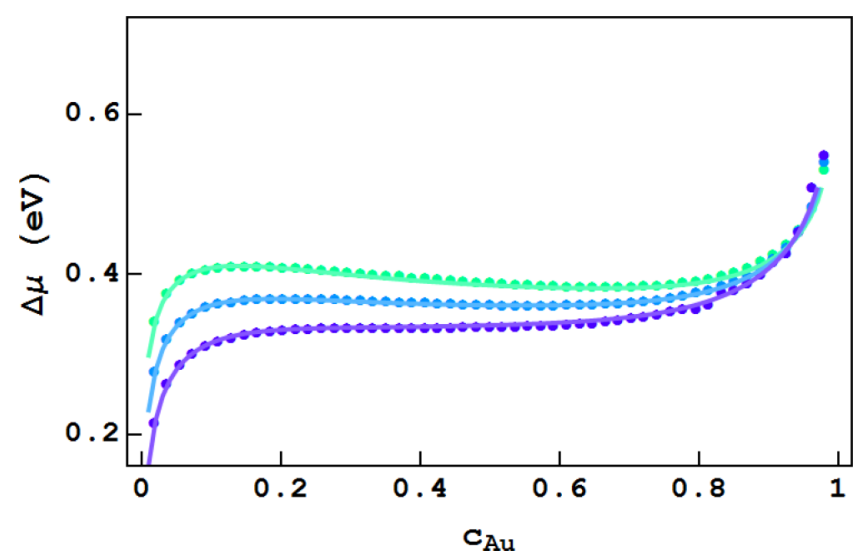

FIG. 9. Isotherms $\Delta \mu(c)$ issued from SMA-MC simulations (points) and ESE-MC simulations taking into account $\Delta S_{\text {vib }}^{\text {perm }}$ (lines) at $T=900 \mathrm{~K}$ (green), $T=1000 \mathrm{~K}$ (blue), and $T=1100 \mathrm{~K}$ (dark blue) for $\mathrm{Au}_{c} \mathrm{Ni}_{1-c}$. 
of atomic positions and homothetic volume changes are allowed [59].

We illustrate the mixed MC mean-field approach in the case of the $\mathrm{Au}_{c} \mathrm{Ni}_{1-c}$ system. From the values $\Delta \mu(c)$, the free energy of permutation is obtained from Eq. (A14) rewritten as follows:

$$
\Delta G^{\mathrm{perm}}=\Delta \mu-k_{B} T \ln \frac{c}{1-c} .
$$

Figure 8(a) shows the $\Delta G^{\text {perm }}(c)$ curves in a range of temperatures for which only a single phase is present, with configurations close to a rss, i.e. when $\alpha_{110} \approx 0$. Here, $\Delta G^{\text {perm }}$ depends linearly on the temperature [Fig. 8(a)], which allows one to evaluate $\Delta S_{\text {vib }}^{\text {perm }}$

$$
\Delta S_{\mathrm{vib}}^{\mathrm{perm}}(c)=-\frac{\Delta G^{\mathrm{perm}}\left(c, T_{2}\right)-\Delta G^{\mathrm{perm}}\left(c, T_{1}\right)}{T_{2}-T_{1}} .
$$

Figure 9(b) shows the evolution of $\Delta S_{\mathrm{vib}}^{\mathrm{perm}}$ as a function of $c$. Here, $\Delta S_{\text {vib }}^{\text {perm }}$ decreases when the concentration increases. As can be seen from the superposition of the curves obtained from the $\Delta G^{\text {perm }}(c)$ curves, the assumption that $\Delta S_{\text {vib }}^{\text {perm }}$ does not depend on the temperature is fully validated. Adding the contribution of the vibrational entropy of permutation in the ESE-MC simulations leads to a perfect agreement with SMA-MC isotherms (see Fig. 9).
[1] P. Olsson, J. Wallenius, C. Domain, K. Nordlund, and L. Malerba, Phys. Rev. B 72, 214119 (2005).

[2] P. L. Williams, Y. Mishin, and J. C. Hamilton, Modell. Simul. Mater. Sci. Eng. 14, 817 (2006).

[3] R. C. Pasianot and L. Malerba, J. Nucl. Mater. 360, 118 (2007).

[4] M. Y. Lavrentiev, R. Drautz, D. Nguyen-Manh, T. P. C. Klaver, and S. L. Dudarev, Phys. Rev. B 75, 014208 (2007).

[5] A. M. Dongare, M. Neurock, and L. V. Zhigilei, Phys. Rev. B 80, 184106 (2009).

[6] G. P. Purja Pun and Y. Mishin, Philos. Mag. 89, 3245 (2009).

[7] G. Bonny, R. Pasianot, D. Terentyev, and L. Malerba, Philos. Mag. 91, 1724 (2011).

[8] M. Briki, Ph.D. thesis, Université Paris Sud (2013).

[9] M. Eich, D. Beinke, and G. Schmitz, Comput. Mater. Sci. 104, 185 (2015).

[10] F. Ducastelle and F. Gautier, J. Phys. F: Met. Phys. 6, 2039 (1976).

[11] F. Ducastelle and G. Tréglia, J. Phys. F: Met. Phys. 10, 2137 (1980).

[12] C. Wolverton and A. Zunger, Comput. Mater. Sci. 8, 107 (1997).

[13] S.-H. Wei, A. A. Mbaye, L. G. Ferreira, and A. Zunger, Phys. Rev. B 36, 4163 (1987).

[14] J. M. Sanchez, J. P. Stark, and V. L. Moruzzi, Phys. Rev. B 44, 5411 (1991).

[15] D. B. Laks, L. G. Ferreira, S. Froyen, and A. Zunger, Phys. Rev. B 46, 12587 (1992).

[16] A. Zunger, in Statics and Dynamics of Alloy Phase Transformations, edited by P. E. A. Turchi and A. Gonis (Plenum, New York, 1994).

[17] D. de Fontaine, Solid State Phys. 47, 33 (1994).

[18] C. Colinet, J. Eymery, A. Pasturel, A. T. Paxton, and M. van Schilfgaarde, J. Phys.: Condens. Matter 6, L47 (1994).

[19] C. Amador and G. Bozzolo, Phys. Rev. B 49, 956 (1994).

[20] Z. W. Lu and A. Zunger, Phys. Rev. B 50, 6626 (1994).

[21] C. Wolverton, V. Ozolins, and A. Zunger, Phys. Rev. B 57, 4332 (1998).

[22] V. Ozolins, C. Wolverton, and A. Zunger, Phys. Rev. B 57, 6427 (1998).

[23] C. Colinet and A. Pasturel, J. Alloys Compd. 296, 6 (2000).

[24] N. A. Zarkevich and D. D. Johnson, Phys. Rev. Lett. 92, 255702 (2004).

[25] M. H. F. Sluiter, C. Colinet, and A. Pasturel, Phys. Rev. B 73, 174204 (2006).
[26] B. Arnold, A. Díaz-Ortiz, G. L. W. Hart, and H. Dosch, Phys. Rev. B 81, 094116 (2010).

[27] J. M. Sanchez, F. Ducastelle, and D. Gratias, Phys. A 128, 334 (1984).

[28] A. van de Walle and G. Ceder, Rev. Med. Phys. 74, 11 (2002).

[29] A. van de Walle, JOM 65, 1523 (2013).

[30] M. Asta and S. M. Foiles, Phys. Rev. B 53, 2389 (1996).

[31] D. A. Papaconstantopoulos and M. J. Mehl, Phys. Rev. B 64 172510 (2001).

[32] M. Yu, D. R. Trinkle, and R. M. Martin, Phys. Rev. B 83, 115113 (2011).

[33] J. Creuze, Ph.D. thesis, Université Paris Sud (2000).

[34] J. Creuze, F. Berthier, and B. Legrand, in Nanoalloys: Synthesis, Structure and Properties (Engineering Materials), edited by D. Alloyeau, C. Mottet, and C. Ricolleau (Springer-Verlag, London, 2012), pp 227-257.

[35] F. Berthier, B. Legrand, and G. Tréglia, Acta Mater. 47, 2705 (1999).

[36] I. Braems, J. Creuze, F. Berthier, R. Tétot, and B. Legrand, Surf. Sci. 602, 1903 (2008).

[37] T. B. Wu and J. B. Cohen, Acta Metall. Mater. 31, 1929 (1983).

[38] M. Bienzle, T. Oishi, and F. Sommer, J. Alloys Compd. 220, 182 (1995)

[39] Ge Meng, R. Claessen, F. Reinert, R. Zimmermann, P. Steiner, and S. Hüfner, J. Phys.: Condens. Matter 8, 5569 (1996).

[40] M. Dynna, A. Marty, B. Gilles, and G. Patrat, Acta Mater. 44, 4417 (1996).

[41] M. Dynna, A. Marty, B. Gilles, and G. Patrat, Acta Mater. 45, 257 (1997).

[42] J.-C. Zhao and M. R. Notis, Metall. Mater. Trans. A 30, 707 (1999).

[43] E. O. Arregui, M. Caro, and A. Caro, Phys. Rev. B 66, 054201 (2002).

[44] S. Labat, F. Bocquet, B. Gilles, and O. Thomas, Scr. Mater. 50, 717 (2004).

[45] H. Reichert, A. Schöps, I. B. Ramsteiner, V. N. Bugaev, O. Shchyglo, A. Udyansky, H. Dosch, M. Asta, R. Drautz, and V. Honkimäki, Phys. Rev. Lett. 95, 235703 (2005).

[46] K. Terakura, T. Oguchi, T. Mohri, and K. Watanabe, Phys. Rev. B 35, 2169 (1987).

[47] L. J. Gallego, J. A. Somoza, J. A. Alonso, and J. M. Lopez, Phys. B 154, 82 (1988).

[48] C. Wolverton, V. Ozolins, and A. Zunger, J. Phys.: Condens. Matter 12, 2749 (2000). 
[49] J. Creuze, F. Berthier, R. Tétot, B. Legrand, and G. Tréglia, Phys. Rev. B 61, 14470 (2000).

[50] I. Meunier, G. Tréglia, R. Tétot, J. Creuze, F. Berthier, and B. Legrand, Phys. Rev. B 66, 125409 (2002).

[51] J. Creuze, F. Berthier, R. Tétot, and B. Legrand, Surf. Sci. 526, 121 (2003).

[52] E. B. Webb, G. S. Grest, D. R. Heine, and J. J. Hoyt, Acta Mater. 53, 3163 (2005).

[53] C. Langlois, D. Alloyeau, Y. Le Bouar, A. Loiseau, T. Oikawa, C. Mottet, and C. Ricolleau, Faraday Discuss. 138, 375 (2008).

[54] V. Rosato, M. Guillopé, and B. Legrand, Philos. Mag. A 59, 321 (1989).

[55] M. Briki, J. Creuze, F. Berthier, and B. Legrand, Solid State Phenom. 172-174, 658 (2011).

[56] C. Kittel, Introduction à la Physique de l'État Solide (Dunod, Paris, 1972).

[57] G. Simmons and H. Wang, Single Crystal Elastic Constants and Calculated Aggregated Properties (MIT Press, Cambridge, MA, 1971).

[58] E. Bitzek, P. Koskinen, F. Gähler, M. Moseler, and P. Gumbsch, Phys. Rev. Lett. 97, 170201 (2006).
[59] K. Binder, The Monte Carlo Method in Condensed Matter Physics (Springer-Verlag, Berlin, 1995).

[60] S. M. Foiles, Phys. Rev. B 32, 7685 (1985).

[61] N. Metropolis, A. Rosenbluth, M. Rosenbluth, and A. H. Teller, J. Chem. Phys. 21, 1087 (1953).

[62] B. Widom, J. Chem. Phys. 39, 2808 (1963).

[63] F. Berthier, J. Creuze, and B. Legrand (unpublished).

[64] H. Amara, J.-M. Roussel, C. Bichara, J.-P. Gaspard, and F. Ducastelle, Phys. Rev. B 79, 014109 (2009).

[65] H. Amara, B. Legrand, and F. Ducastelle (unpublished).

[66] M. J. Portmann, B. Schönfeld, G. Kostorz, and F. Altorfer, Phys. Rev. B 65, 024110 (2001).

[67] M. J. Portmann, B. Schönfeld, G. Kostorz, F. Altorfer, and J. Kohlbrecher, Phys. Rev. B 68, 012103 (2003).

[68] H. Hokamoto and T. B. Massalski, Binary Alloy Phase Diagrams (American Society for Metals, Metals Park, OH, 1986).

[69] F. Ducastelle, Order and Phase Stability in Alloys (NorthHolland, Amsterdam, 1991).

[70] F. Ducastelle, B. Legrand, and G. Tréglia, Prog. Theor. Phys. Suppl. 101, 159 (1990).

[71] M. E. Fisher, Rep. Prog. Phys. 30, 615 (1967). 